\title{
High levels of maternal vitamin D may be protective against multiple sclerosis
}

Vitamin D intake has previously been linked to the etiology of multiple sclerosis (MS). Now, Fariba Mirzaei and her colleagues have shown that gestational levels of vitamin D might also confer a protective effect against the disease in the offspring.

\section{Mothers with higher dietary vitamin D intake ... had daughters with a lower risk of MS 77}

Vitamin D is one of many genetic and environmental factors that are associated with MS. Epidemiological studies of childhood sun exposure, dietary vitamin $\mathrm{D}$ intake and serum levels of 25-hydroxyvitamin D have indicated that higher levels are protective against the disease. Some evidence has also indicated that gestational levels of vitamin D might also confer a protective effect; there is greater concordance in MS rates among dizygotic twins, who share the same intrauterine environment, than in nontwin siblings, and season of birth is linked to MS risk in a manner that suggests that maternal sun exposure reduces disease risk in the offspring. However, the relationship between gestational vitamin $\mathrm{D}$ levels and MS has not, until now, been directly studied.

In a large longitudinal study Mirzaei et al. measured the association between exposure to vitamin $\mathrm{D}$ during gestation and risk of MS in adulthood. The researchers used data from 35,000 mothers and daughters participating in the Nurses' Health and Nurses' Mothers' Studies. Individuals provided demographic, lifestyle and health-related information biennially, and their mothers provided information about their experiences during pregnancy and the early-life exposures of their daughters. Maternal vitamin $\mathrm{D}$ exposure was measured by milk intake, dietary vitamin $\mathrm{D}$ intakebased on reports of foods eaten during pregnancy-and serum 25-hydroxyvitamin D levels-calculated using a prediction model-during pregnancy.

The researchers found a $38 \%$ lower risk of MS in the daughters of mothers who drank two or three glasses of milk per day than in the daughters of those who drank little or no milk. Mothers with higher dietary vitamin D intake and preditcted serum vitamin D levels also had daughters with a lower risk of MS.

The protective effect of maternal milk intake could be mediated by vitamin $\mathrm{D}$, but other nutrients might be involved. However, taken together with the results from dietary and predicted serum vitamin D measures, and in light of previous research, this study indicates that gestational exposure to vitamin $\mathrm{D}$ is protective against the later development of MS.

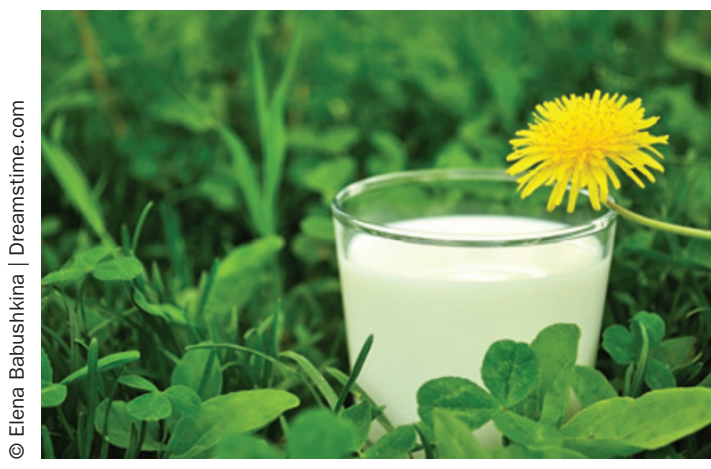

The mechanism by which vitamin $\mathrm{D}$ confers its protective effect requires further investigation, and the authors conclude that these results should be interpreted cautiously until they have been verified in other populations. They also plan to develop analyses involving biomarkers of vitamin D status in pregnancy. Although the role of gestational vitamin $\mathrm{D}$ is not certain, advising pregnant women to increase their vitamin D intake is not inherently risky and, as Mirzaei et al. state, "some cases of MS could be prevented by increasing exposure to vitamin D during intrauterine life."

Eleanor Beal

Original article Mirzaei, F. et al. Gestational vitamin D and the risk of multiple sclerosis in the offspring. Ann. Neurol. doi:10.1002/ana.22456 